\title{
Epidemiology of Giardia duodenalis infection in ruminant livestock and children in the Ismailia province of Egypt: insights by genetic characterization
}

Yosra A. Helmy ${ }^{1,2^{*}{ }^{+}}$, Christian $\mathrm{Klotz}^{3 \dagger}{ }^{+}$, Hendrik Wilking ${ }^{4}$, Jürgen Krücken ${ }^{5}$, Karsten Nöckler ${ }^{6}$, Georg Von Samson-Himmelstjerna ${ }^{5}$, Karl-H. Zessin ${ }^{2}$ and Toni Aebischer ${ }^{3}$

\begin{abstract}
Background: Giardia duodenalis is a common flagellated protozoan parasite that infects the small intestine of a wide range of vertebrate hosts. This study aimed to determine whether tracing of $\mathrm{G}$. duodenalis isolates by current genetic typing tools is possible using an exemplary set of samples from infected cattle, buffalo and children from the Ismailia province, Egypt.

Method: A total of 804 fecal samples from ruminant animals was collected from 191 herds and 165 samples from diarrheal children below the age of 10 years. Parasites were detected in these samples using the copro-antigen RIDA ${ }^{\circledR}$ QUICK test and by real-time PCR. Samples were then genetically characterized based on the triosephosphate isomerase, glutamate dehydrogenase and $\beta$-giardin genes.

Results: The prevalence of $G$. duodenalis was $53 \%$ in ruminants and $21 \%$ in symptomatic children and infection was not positively correlated with diarrheal symptoms. Sequence typing analysis confirmed predominance of B-type sequences $(>67 \%)$ in humans and E-type sequences (>81\%) in ruminants over A-type sequences. For 39 samples the complete sequence information of the three marker gene fragments could be derived. Integration of the concatenated sequence information of the three marker gene fragments with the spatial data of the respective sample revealed that identical or near identical (only up to 1 out of 1358 bp different) concatenated sequencing types were spatially related in 4 out of 5 cases.

Conclusion: The risk of zoonotic infection emanating from ruminants even in high prevalence areas is negligible. Genetic characterization indicated a predominant anthropogenic cycle of infection within the pediatric population studied. Integration of sequence typing data with information on geographic origins of samples allows parasite sub-population tracing using current typing tools.
\end{abstract}

Keywords: Giardia, Genetic characterization, Ruminants, Diarrheal children, Real time PCR, RIDA®QUICK test, Triose phosphate isomerase (tpi), Glutamate dehydrogenase (gdh), $\beta$-giardin (bg), Spatial distribution, Egypt

\footnotetext{
* Correspondence: yosra.mohamed@fu-berlin.de

${ }^{\dagger}$ Equal contributors

'Department of Animal Hygiene, Zoonoses and Animal Ethology, Faculty of

Veterinary Medicine, Suez Canal University, 41511 Ismailia, Egypt

${ }^{2}$ Faculty Panel Veterinary Public Health, Freie Universität Berlin, 14163 Berlin,

Germany

Full list of author information is available at the end of the article
}

\section{Biomed Central}

(c) 2014 Helmy et al.; licensee BioMed Central Ltd. This is an Open Access article distributed under the terms of the Creative Commons Attribution License (http://creativecommons.org/licenses/by/2.0), which permits unrestricted use, distribution, and reproduction in any medium, provided the original work is properly credited. The Creative Commons Public Domain Dedication waiver (http://creativecommons.org/publicdomain/zero/1.0/) applies to the data made available in this article, unless otherwise stated. 


\section{Background}

Giardia duodenalis (syn. G. lamblia and G. intestinalis) is one of the most frequent enteroparasites worldwide and has been included in the WHO 'neglected disease initiative' [1]. In particular, children in resource-poor countries can be severely affected by G. duodenalis infections, which may lead to significant malabsorption, weight loss and growth retardation [1,2]. The gastrointestinal manifestations include diarrhea, abdominal cramps, greasy stools, flatulence, epigastric tenderness, and steatorrhea accompanied by full-blown malabsorption syndrome [3]. However, infection that is not associated with such overt symptoms occurs frequently, especially in resource-poor countries [4]. These infections may often go unnoticed but epidemiological observations suggest that they are still associated with a malabsorption phenotype [5].

Transmission of Giardia cysts to humans occurs mainly via ingestion of contaminated water or food but parasites can also be directly contracted from infected individuals [6]. In resource-poor countries Giardia is still mainly diagnosed by light microscopy and detection of either trophozoites or cysts in fecal samples. This technique suffers from low sensitivity in comparison to improved methods based on immunodiagnostic fecal antigen detection or polymerase chain reaction (PCR) [7-9]. The latter assays are thus more useful for epidemiological studies $[5,9,10]$, in particular in combination with genetic characterization of the parasites [6]. It is well established that G. duodenalis represents a species complex composed of at least eight genetic groups (assemblages A to $\mathrm{H}$ ) that are distinguishable based on genetic polymorphisms in, e.g., the triosephosphate isomerase (tpi), glutamate dehydrogenase $(g d h)$, beta-giardin $(b g)$ and small-subunit rDNA (ssu-rDNA) genes [6,11-15]. Typing according to these markers is relatively robust and to date has revealed limited evidence for inter-assemblage recombination [16]. If it did occur, such recombination would likely have a tremendous effect on parasite host ranges since assemblages differ in their host specificity, with assemblages $\mathrm{A}$ and $\mathrm{B}$ showing the broadest host range infecting humans and numerous other mammals including companion animals and livestock [15,17-19]. G. duodenalis is considered potentially zoonotic, largely due to the host distribution of A- and B-type parasites $[15,18,19]$. However, the impact, if any, of zoonotic transmission in countries with low or high prevalence remains unclear [15].

The scope of the present study was to improve the understanding of the epidemiology of G. duodenalis infections in the Ismailia province in Egypt. Therefore, the prevalence of Giardia infections in children with diarrheal symptoms and in ruminants (cattle and buffalo) was determined. Infection was assessed using an immunodiagnostic, copro-antigen detection test (RIDA $\left.{ }^{\circ} \mathrm{QUICK}\right)$ and a real time PCR- assay for detection of Giardia
ssu-rDNA. In addition, parasite DNA was genetically characterized based on $t p i, g d h$ and $b g$ genes to elucidate distribution of $G$. duodenalis types, and to gather evidence for transmission of Giardia from ruminants to humans in this area.

\section{Methods}

\section{Ethics statement}

The study was reviewed and approved by the University Board, Suez Canal University. Approval included the use of oral consent (human and animal samples) due to low literacy rates. Oral consent of the parents or legal guardians of the children was obtained in each case and was witnessed by a hospital physician or chief nurse. Privacy and confidentiality were assured. The oral consent was documented in the patient's medical record in the hospital. All samples were non-invasive (only fecal samples) and were analyzed anonymously. Fecal samples from animals were collected rectally after approval from their owners and assurance of anonymity, witnessed by a veterinarian from the Egyptian Veterinary Medicine Authority.

\section{Study area and sample collection}

The study area was the Ismailia province of Egypt from April to June 2011. The Ismailia arable belt runs along the Suez Canal and is one of the most densely populated provinces in Egypt with regard to livestock and people [20]. For $98 \%$ of the farms, farmers either do not own agricultural land or holdings are very small $(<0.4$ ha; herd sizes $<10$; low producing native or crossbred animals) with mixed herds of cattle and buffalo. Animals in the smallholder system are kept in the home compounds and are fed with crop by-products. They have access to surface water or water from shallow wells [20].

The survey considered the distribution of animal holdings over the districts of the province, and aimed to collect fecal samples from animals of a proportional number of the predominantly smallholder farms per district. Samples were mixed with an equal amount of $5 \%$ potassium dichromate and kept at $4{ }^{\circ} \mathrm{C}$ until examination.

A total of 804 animal fecal samples were collected from 191 herds, 593 samples from cattle (246 males and 347 females) and 211 samples from buffalo (83 males and 128 females). Sampling included diarrheal and nondiarrheal animals, with an emphasis on calves. Each animal was identified using a specific system: nomenclature consists of the herd followed by the animal number. For example, 31b/C7 represents cattle 7 ( $\mathrm{C}$ for cattle, $\mathrm{B}$ for buffalo) of the herd $\mathrm{b}$ at location 31 .

Concurrently, stool samples from 165 children up to 10 years of age were collected [20]. Of these, 136 were from diarrheal children hospitalized in one of the province's 9 district hospitals, with 10 to 23 stool samples 
per hospital. 29 were from diarrheal children living on farms where animal samples were also collected.

Attributes of animals (type of drinking water and type of soil available) and children (type of drinking water, place of residence and animal contact) were recorded. The prevalence of Cryptosporidium spp. in this sample population was reported in an earlier study [20].

\section{Giardia diagnostics}

All samples were examined using the copro-antigen RIDA ${ }^{\circ}$ QUICK Cryptosporidium/Giardia Combi (N1122) kit (R-BIOPHARM AG, Darmstadt, Germany) as a screening test, following the manufacturer's instructions with $100 \mu \mathrm{l}$ of the liquid fecal sample. According to the information provided by the supplier, the screening test (for fresh human samples) has 100\% sensitivity, 95.2\% specificity, $88.2 \%$ positive predictive value, and $100 \%$ negative predictive value compared to conventional microscopy that was used as gold standard.

Animal samples that were positive in the $R I D A^{\circ} \mathrm{QUICK}$ test and $10 \%$ of randomly selected negative samples [21] were tested further using Giardia-specific real-time PCR [22]. All human samples were subjected to RIDA $^{\circ} \mathrm{QUICK}$ test and Giardia-specific real-time PCR. For the Giardiaspecific real-time PCR a previously established protocol was applied [9,22] using the Maxima Probe qPCR Master Mix (Thermo Scientific) with $1 \mu \mathrm{l}$ of the target DNA in a total volume of $25 \mu \mathrm{l}$. Amplification (40 PCR cycles), detection, and data analysis were performed using an Mx3000P cycler and MxPro software (Stratagene). Analytical specificity was $94.1 \%$ ( 1 of 17 non-template-controls was weakly positive, $c t=38.9$ ). Analytical sensitivity was determined using a calibrated parasite DNA solution and was equivalent to the detection of 1 parasite $(1$ trophozoite $=4 n$ ) giving a ct $=39.8$.

\section{DNA extraction}

Genomic DNA was extracted from $1 \mathrm{ml}$ of sample using the NucleoSpin ${ }^{\bullet}$ Soil kit (Macherey-Nagel, Germany) and using an elution volume of $60 \mu$ l. Extracted DNA was analyzed by agarose gel electrophoresis and stored at $-20^{\circ} \mathrm{C}$ until further examinations. DNA concentration and purity were assessed spectrophotometrically.

\section{Genetic characterization of Giardia isolates}

Genetic characterization of Giardia isolates was performed using previously described nested PCR protocols to amplify fragments of the tpi- [13], $g d h$ [12]- and $b g$ [14,23]-gene loci. Some of the samples were also analyzed for assemblage A- and E-specific tpi-gene fragments as previously published [24]. As a standard procedure, 1-3 $\mu \mathrm{l}$ of sample DNA were used for the primary PCR and $1-2 \mu \mathrm{l}$ of the primary PCR reaction were applied to the nested PCR. All PCR reactions were performed using $2.5 \mathrm{U}$ of the
MangoTaq ${ }^{\mathrm{Tx}}$ DNA Polymerase and the according buffer system (Bioline).

PCR reactions were analyzed by agarose gel electrophoresis and PCR products were purified using the DNA Clean\&Concentrator ${ }^{\text {Tn }}$ kit (ZYMO RESEARCH). Sequencing reactions were performed using Big dye 3.1 sequencing reagents (Applied biosystems) and applied to the RKI in-house sequencing facility. The obtained sequences were analyzed to identify the most similar sequence deposited in public databases by applying the Basic Local Alignment Search Tool (BLAST) (http://blast.ncbi.nlm.nih.gov/Blast.cgi) using the nucleotide collection (nr/nt) database in April 2013. The sequences were also compared to dedicated reference sequences [25] in order to identify the assemblage and subassemblage level. Therefore, alignments of single and in silico concatenated genes were produced using MUSCLE [26] or ClustalW integrated subroutines of Geneious version 6.1.6 (Biomatters). Sequence relatedness was analyzed by distance comparison between sequences based on the number of identical nucleotide residues. See Additional file 1: Table S1 for the accession numbers of the reference sequences used for the analysis. For spatial analysis sequences that were identical or that differed only in one nucleotide were combined in 'clusters' and sample distribution was visualized onto the map of the Ismailia province using the program ArcGIS 9.5 (ESRI).

\section{Nucleotide sequence accession numbers}

Nucleotide sequences generated in this study have been deposited into the GenBank database under accession numbers [GenBank: KF957627-KF957634, KJ124862-KJ124918, KJ124983-KJ125044, KJ124919-KJ124982].

\section{Statistical analysis}

Statistical analysis was performed using mid-P exact probability tests and differences were considered significant when $p$-values $\leq 0.05$ were obtained (http://www.openepi. com/v37/Menu/OE_Menu.htm). The real-time PCR-based Giardia prevalence in animal samples was estimated by re-testing a random selection of $10 \%$ of samples negative in the rapid screening test and extrapolating the data to the whole animal sample population [21].

\section{Results}

Estimation of the prevalence of Giardia infections in humans and accompanying livestock

Copro-antigen tests are attractive for epidemiological surveys of the burden of gastrointestinal infections since they are highly processive. Here the $R I D A^{\circ} \mathrm{QUICK}$ test was used to quickly evaluate Giardia prevalence in all samples collected for this survey. In total, 13 out of 165 (8\%) samples from diarrheal children and 50 out of 804 (6\%) animal samples tested positive. However, based 
solely on antigen-detection tests, prevalence can be significantly underestimated (although less than when based on classical microscopic tests; $[5,7,27])$. Therefore, a more sensitive strategy based on a real-time PCR assay [9,22] directed to the multi-copy parasite ssu-rDNA in fecal samples was designed to obtain a more accurate estimate of the prevalence. To this end, all human samples were reanalyzed and, to estimate prevalence in animals, samples of all copro-antigen test positive ruminants were re-analyzed as well as a randomly chosen $10 \%$ of the copro-antigen test negative animal samples.

In children with diarrhea, results from real-time PCR revealed an overall Giardia prevalence of 21\% (Table 1). The age dependent prevalence varied but the highest value (29\%) was observed in children up to 1 year old

Table 1 Estimation of Giardia duodenalis prevalence in diarrheal children

\begin{tabular}{|c|c|c|}
\hline \multirow[b]{2}{*}{ Age group } & \multicolumn{2}{|c|}{$\begin{array}{c}\text { Giardia prevalence in percent } \\
\text { (Number of samples: positive/total) } \\
\text { (95\% confidence interval) }\end{array}$} \\
\hline & $\begin{array}{l}\text { Copro-antigen test } \\
\left(R I D A^{\oplus} Q^{U} \text { UICK) }\right.\end{array}$ & Real time PCR assay \\
\hline \multirow[t]{4}{*}{ up to $1 y$} & 0 & 29 \\
\hline & $(0 / 24)$ & $(7 / 24)$ \\
\hline & $(0-11.7)$ & $(13.7-49.4)$ \\
\hline & & Ct value: $38.4(33.2-39.0)$ \\
\hline \multirow[t]{4}{*}{$>1 y-2 y$} & 9 & 26 \\
\hline & $(3 / 35)$ & $(9 / 35)$ \\
\hline & $(2.2-21.6)$ & $(13.3-41.9)$ \\
\hline & & Ct value: 34.5 (26.1 - 38.0) \\
\hline \multirow[t]{4}{*}{$>2 y-3 y$} & 6 & 16 \\
\hline & $(2 / 32)$ & $(5 / 32)$ \\
\hline & $(1.1-19.2)$ & $(5.9-31.3)$ \\
\hline & & Ct value: 30.7 (18.7 - 37.4) \\
\hline \multirow[t]{4}{*}{$>3 y-4 y$} & 10 & 24 \\
\hline & $(2 / 21)$ & $(5 / 21)$ \\
\hline & $(1.6-28.1)$ & $(9.3-45.2)$ \\
\hline & & Ct value: 33.8 (29.3 - 39.3) \\
\hline \multirow[t]{4}{*}{$>4 y-10 y$} & 11 & 17 \\
\hline & $(6 / 53)$ & $(9 / 53)$ \\
\hline & $(4.7-22.1)$ & $(8.6-28.9)$ \\
\hline & & Ct value: 29.0 (22.7 - 39.6) \\
\hline \multirow[t]{4}{*}{ Total } & $8^{\mathrm{a}}$ & $21^{\mathrm{b}}$ \\
\hline & $(13 / 165)$ & $(35 / 165)$ \\
\hline & $(4.5-12.8)$ & $(15.5-28)$ \\
\hline & & Ct value: $33.2(18.7-39.6)$ \\
\hline
\end{tabular}

Different superscripts $(a, b)$ : significant difference at $p<0.05$. Median ct values (range) of real time PCR assay for assigned age groups are shown. (not significant). Interestingly, the real-time PCR assays on samples from these infants yielded comparatively high ct values indicating lower parasite DNA quantities relative to samples from older children (Table 1). All samples in this infant age group that were tested positive by real-time PCR were negative in the copro-antigen test. A comparison of median real-time PCR ct values from samples positive in both the copro-antigen test and by real-time PCR, versus samples only positive by real time PCR suggested a significantly higher abundance ( 120 fold) of Giardia DNA in copro-antigen test-positive samples ( median $\mathrm{ct}=28.1$ vs. $=35.1, \mathrm{p}<0.05$, Mann Whitney test).

In animals, all positive copro-antigen tests were confirmed by real-time PCR. In addition, $50 \%$ of the subset of copro-antigen negative samples were also positive. Thus, a value for total prevalence of $53 \%$ in the sampled livestock was extrapolated (Table 2). The age-specific prevalence estimate ranged between 50-57\%, with a trend for higher values in young calves (1 day- 3 months, not significant) (Table 2). Moreover, testing positive for Giardia in either the copro-antigen or the real-time PCR based test was not correlated with diarrhea in these animals (data not shown and Table 3).

\section{Risk factors for contracting Giardia infection}

Occurrence of Giardia was analyzed for associations with potential risk factors determined for the two sampled populations. For humans, gender, residency status, farm animal contact, source of drinking water and co-infection with Cryptosporidium spp. (a second locally highly prevalent zoonotic protozoan that causes gastrointestinal disease) were investigated.

The prevalence of Cryptosporidium spp. infections amongst the studied cattle, buffalo and pediatric populations has been recently reported by our team [20]. Here, Giardia-Cryptosporidium spp. co-infections detected by real-time PCR were observed in 36\% of animals and 11\% of children. Assuming that co-infection will happen by chance, its prevalence can be estimated based on the product of the prevalence of individual infections. Accordingly, the expected prevalence of co-infection was calculated to be $17 \%$ in animals and $10 \%$ in children. Thus, the value in humans corresponded well with the observed numbers, suggesting neither positive nor negative effects of one infection on the risk of contracting the other pathogen. In animals, however, the calculated value was less than half of the observed prevalence ( $\mathrm{p}<0.05$, Fisher's exact test) of coinfection implying a factor that promotes Cryptosporidium and Giardia co-infections.

For the pediatric population under study, gender, residency status and contact with livestock did not correlate with Giardia infection. However, a nearly 3-fold higher probability of Giardia infection was associated with drinking tap water (Table 4). 
Table 2 Estimation of Giardia duodenalis prevalence in cattle and buffalo

\begin{tabular}{|c|c|c|c|c|c|}
\hline \multirow[b]{3}{*}{ Age group } & \multicolumn{5}{|c|}{$\begin{array}{c}\text { Giardia prevalence in percent } \\
\text { (Number of samples: positive/total) } \\
\text { ( } 95 \% \text { confidence interval) }\end{array}$} \\
\hline & \multicolumn{3}{|c|}{ Copro-antigen test (RIDA $\left.{ }^{\circledR} Q U I C K\right)$} & \multicolumn{2}{|c|}{ Real time PCR assay ${ }^{b}$} \\
\hline & $\overline{\text { All }}$ & Cattle & Buffalo & $\begin{array}{l}10 \% \text { of quick test } \\
\text { negative samples }\end{array}$ & $\begin{array}{l}\text { Total estimated } \\
\text { prevalence }\end{array}$ \\
\hline \multirow[t]{3}{*}{$1 d-1 m$} & 9 & 9 & 8 & 52 & 57 \\
\hline & $(22 / 244)$ & $(17 / 183)$ & $(5 / 61)$ & $(12 / 23)$ & $(138 / 244)$ \\
\hline & $(5.9-13.1)$ & $(5.7-14.2)$ & $(3.1-17.2)$ & $(32.1-71.7)$ & $(50.3-62.7)$ \\
\hline \multirow[t]{3}{*}{$>1-3 \mathrm{~m}$} & 5 & 5 & 4 & 53 & 55 \\
\hline & $(5 / 103)$ & $(4 / 79)$ & $(1 / 24)$ & $(8 / 15)$ & $(57 / 103)$ \\
\hline & $(1.8-10.4)$ & $(1.6-11.8)$ & $(0.2-18.9)$ & $(28.7-76.8)$ & $(45.7-64.7)$ \\
\hline \multirow[t]{3}{*}{$>4-6 \mathrm{~m}$} & 6 & 7 & 2 & 46 & 50 \\
\hline & $(9 / 149)$ & $(8 / 107)$ & $(1 / 42)$ & $(6 / 13)$ & $(74 / 149)$ \\
\hline & $(2.9-10.8)$ & $(3.5-13.7)$ & $(0.11-11.2)$ & $(21.3-72.6)$ & $(41.7-57.7)$ \\
\hline \multirow[t]{3}{*}{$>6 \mathrm{~m}$} & 4 & 5 & 4 & 48 & 50 \\
\hline & $(14 / 308)$ & $(11 / 224)$ & $(3 / 84)$ & $(12 / 25)$ & $(155 / 308)$ \\
\hline & $(2.6-7.3)$ & $(2.6-8.4)$ & $(0.9-9.4)$ & $(29.2-67.3)$ & $(44.8-55.9)$ \\
\hline \multirow[t]{3}{*}{ Total } & 6 & 7 & 5 & 50 & 53 \\
\hline & $(50 / 804)$ & $(40 / 593)$ & $(10 / 211)$ & $(38 / 76)$ & $(424 / 804)$ \\
\hline & $(4.7-8.1)$ & $(4.9-8.9)$ & $(2.4-8.3)$ & $(38.9-61.1)$ & $(49.3-56.2)$ \\
\hline
\end{tabular}

${ }^{\mathrm{b}}$ All copro-antigen test positives and $10 \%$ of the copro-antigen negative samples were analyzed by real time PCR. Prevalence was calculated by extrapolation assuming that the analyzed negative samples are representative for all negative samples. Note that all positive copro-antigen tests were confirmed by real time PCR assay. Note: No significant differences were detected between the age groups.

Genetic characterization of Giardia suggests rare zoonotic transmission from livestock to humans

Copro-antigen detection and the chosen ssu-rDNA real-time PCR assay do not provide information about the sub-species or assemblage of Giardia present in a sample. The latter information, however, is necessary to assess likely transmission cycles. Thus, all samples that were applied to the real-time PCR assay were subjected to genetic characterization at the tpi-, gdh-and $b g$-gene loci [12-14]. Overall, parasite DNA from 90 samples (22 human and 78 animal samples) could be characterized at least at one locus (Additional file 2: Table S2). Of these, 52 samples

Table 3 Prevalence of Giardia duodenalis in animal samples in relation to fecal consistency

\begin{tabular}{|c|c|c|c|c|c|c|c|c|}
\hline & \multicolumn{8}{|c|}{$\begin{array}{l}\text { Number of Giardia cases } \\
\text { (percent) } \\
\text { (95\% confidence interval) }\end{array}$} \\
\hline & \multicolumn{4}{|c|}{ Copro-antigen (RIDA $\left.{ }^{\oplus} Q U I C K\right)$ test } & \multicolumn{4}{|c|}{ Real time PCR assay ${ }^{+}$} \\
\hline & Total & Watery & Pasty & Normal & Total & Watery & Pasty & Normal \\
\hline \multirow[t]{3}{*}{ Positive } & 50 & 20 & 21 & 9 & 88 & 37 & 36 & 15 \\
\hline & (6) & $\left(40^{*}\right)$ & $\left(42^{*}\right)$ & $\left(18^{*}\right)$ & (70) & $\left(42^{*}\right)$ & $\left(41^{*}\right)$ & $\left(17^{*}\right)$ \\
\hline & $(5-8)$ & $(27-54)$ & $(29-56)$ & $(9-30)$ & $(61-77)$ & $(32-53)$ & $(31-51)$ & $(10-26)$ \\
\hline \multirow[t]{3}{*}{ Negative } & 754 & 254 & 284 & 216 & 38 & 12 & 21 & 5 \\
\hline & (94) & $\left(34^{*}\right)$ & $\left(38^{*}\right)$ & $\left(29^{*}\right)$ & (30) & $\left(32^{*}\right)$ & $\left(55^{*}\right)$ & $\left(13^{*}\right)$ \\
\hline & $(92-95)$ & $(30-37)$ & $(34-41)$ & $(26-32)$ & $(22-38)$ & $(18-48)$ & $(39-70)$ & $(5-27)$ \\
\hline \multirow[t]{3}{*}{ Total } & 804 & 274 & 305 & 225 & 126 & 49 & 57 & 20 \\
\hline & & (34) & (38) & (28) & & (39) & (45) & (16) \\
\hline & & $(31-37)$ & $(35-41)$ & $(25-31)$ & & $(31-48)$ & $(37-54)$ & $(10-23)$ \\
\hline
\end{tabular}

${ }^{+}$Only experimental results are presented.

*Percentage calculated as proportion of Giardia positive and negative samples, respectively.

Note: No significant differences of fecal consistency were detected of Giardia positive and Giardia negative animals. 
Table 4 Analysis of potential risk factors for Giardia infections in diarrheal children

\begin{tabular}{|c|c|c|c|c|c|c|c|c|}
\hline & \multicolumn{8}{|c|}{$\begin{array}{l}\text { Risk factor dependent Giardia infection in percent } \\
\text { (Number of Giardia cases: positive/total) } \\
\text { (95\% confidence interval) }\end{array}$} \\
\hline & \multicolumn{2}{|c|}{ Gender } & \multicolumn{2}{|c|}{ Residency } & \multicolumn{2}{|c|}{ Contact with animals } & \multicolumn{2}{|c|}{ Source of water } \\
\hline & Male & Female & Village & City & Contact & No contact & Underground & Tap \\
\hline \multirow[t]{3}{*}{ Copro-antigen test } & 9.5 & 6.2 & 9.0 & 7.0 & 9.0 & 5.0 & $5.0^{*}$ & $9.0^{*}$ \\
\hline & $(8 / 84)$ & $(5 / 81)$ & $(7 / 82)$ & $(6 / 83)$ & $(10 / 108)$ & $(3 / 57)$ & $(3 / 57)$ & $(10 / 108)$ \\
\hline & $(4.5-17.3)$ & $(2.3-13.2)$ & $(3.8-16.2)$ & $(2.9-14.4)$ & $(4.8-15.9)$ & $(1.4-13.7)$ & $(1.4-13.7)$ & $(4.8-15.9)$ \\
\hline \multirow[t]{3}{*}{ Real time PCR assay } & 22.6 & 19.8 & 23.0 & 19.0 & 21.0 & 21.0 & $11.0^{+}$ & $27.0^{+}$ \\
\hline & $(19 / 84)$ & $(16 / 81)$ & $(19 / 82)$ & $(16 / 83)$ & $(23 / 108)$ & $(12 / 57)$ & $(6 / 57)$ & $(29 / 108)$ \\
\hline & $(14.6-32.5)$ & $(12.2-29.5)$ & $(15-33.2)$ & $(11.9-28.8)$ & $(14.4-29.8)$ & $(11.9-33)$ & $(4.4-20.6)$ & $(19-35.8)$ \\
\hline
\end{tabular}

${ }^{*} p=0.390 ;{ }^{+} p=0.013$

could be typed at all three loci and 14 samples at two loci. Repeating the typing assays of the negative samples did not improve this yield (each locus-specific PCR was repeated at least once if a sample was negative in the first attempt). Genetic characterization yielded the following qualitative and quantitative results:

Firstly, typing produced previously observed ambiguities [6]. Typing of eight samples was ambiguous in the sense that sequences obtained for a particular locus or for different loci had to be assigned to different assemblages (Table 5). In another group of samples a different kind of expected ambiguity was observed, namely, double peaks at specific sites in the electropherograms of the sequencing reactions. Such peak patterns were observed in three assemblage A type, 15 B type and $39 \mathrm{E}$ type sequences. Several of these patterns were repeatedly observed in different samples (Additional file 1: Table S1).

Secondly, typing of Giardia DNA present in human samples confirmed that assemblage B is more frequently found than assemblage A (Table 6). Assemblage B was assigned based on tpi to $69 \%$, on $g d h$ to $67 \%$, and on $b g$

Table 5 Samples assigning for different G. duodenalis assemblages at different gene loci

\begin{tabular}{lllllll}
\hline Sample ID & Host & tpi & $\begin{array}{l}\text { Ass. A } \\
\text { specific tpi }\end{array}$ & $\begin{array}{l}\text { Ass. E } \\
\text { specific tpi }\end{array}$ & bg & gdh \\
\hline $6 / C 3$ & Cattle & A & A & - & E & E \\
$42 \mathrm{a} / \mathrm{C} 3$ & Cattle & A & A & E & E & E \\
$21 \mathrm{~b} / \mathrm{C} 19$ & Cattle & E & - & - & E & A \\
$19 \mathrm{a} / \mathrm{C} 3$ & Cattle & E & - & - & $\mathrm{A}$ & - \\
$\mathrm{H} 135$ & Human & - & - & - & $\mathrm{A} / \mathrm{E}$ & $\mathrm{E}$ \\
$31 \mathrm{a} / \mathrm{C} 4$ & Cattle & $\mathrm{A} / \mathrm{E}$ & - & $\mathrm{E}$ & $\mathrm{E}$ & $\mathrm{E}$ \\
$\mathrm{H} 86$ & Human & $\mathrm{B}$ & $\mathrm{A}$ & - & $\mathrm{B}$ & $\mathrm{A} / \mathrm{B}$ \\
$15 \mathrm{~d} / \mathrm{C} 10$ & Cattle & - & - & $\mathrm{E}$ & - & $\mathrm{A} / \mathrm{E}$ \\
\hline
\end{tabular}

$(-)=$ negative result.

Note: All PCRs and sequence analysis were performed at least twice with similar results. to $69 \%$ of the samples. Noteworthy, DNA from two human samples (samples H31 and H135) produced assemblage E type sequences at the $g d h$ locus implying infection of children with a livestock assemblage (Table 6, Additional file 2: Table S2). For sample H135 results at the $g d$ locus identified assemblage A in the sample most likely indicative of a mixed infection (Table 5). For H31 no other locus could be typed.

Thirdly, the dominance of assemblage $\mathrm{E}$ type sequences in ruminants was also confirmed [4,28] (Table 6). However, proportions assigned to assemblage $\mathrm{E}$ were variable depending on the locus analyzed ( $81 \%$ on the tpi locus, $94 \%$ on $g d h$ and $98 \%$ on $b g$ loci) (Table 6). Of note, typing efficiency of PCR at the different loci was $60 \%(t p i), 57 \%$ $(g d h)$ and $51 \%(b g)$, respectively. Fourteen of 15 non-E type sequences deduced from animal samples belonged to assemblage A with 11 of these 14 detected by the tpi typing assay. Two of the A type tpi sequences found in animals (samples 10/C11 and 54b/C7, Additional file 2: Table S2) were identical to sub-assemblage AI and AII reference sequences, respectively. Interestingly, these were originally described for parasites isolated from human cases [17]. Unfortunately, complementing the typing of these samples at the other loci failed (Additional file 2: Table S2).

In summary, assemblage distribution within the host species investigated was consistent with expectations, as were assemblage- and locus-specific sequence polymorphisms [29].

Relatedness and spatial distribution of sequencing types Multi locus sequence typing data together with information on the geographic origin of isolates is increasingly used to understand the distribution and dynamics of pathogen populations or individual subgroups in space and time (c.f. http://www.spatialepidemiology.net/; [30]). Here, this approach was adopted to test if analysis of the genetic structure of the parasite population derived from the three loci typing scheme can yield epidemiologically 
Table 6 Type and number of G. duodenalis assemblages detected in animal and human samples by multi locus sequence typing

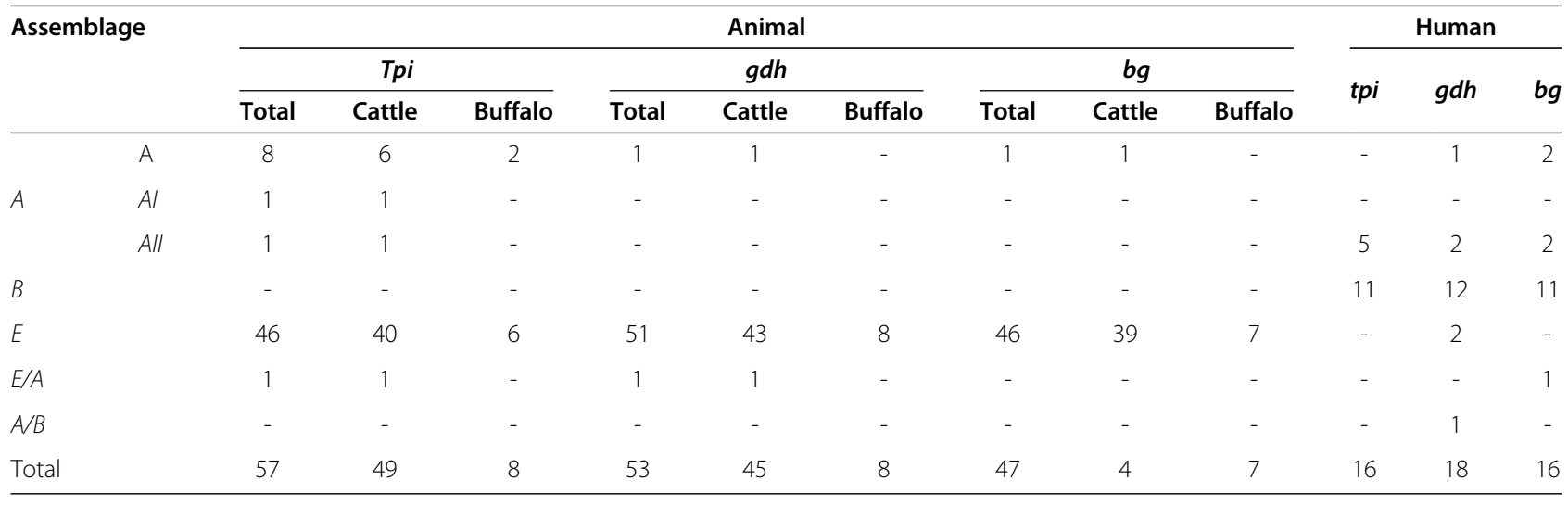

relevant information. To this end, the potential relatedness of parasite DNA types obtained from human or animal samples was analyzed and then checked for its correspondence to the spatial distribution of the respective samples.

Full sequencing information with complete sequence of both strands of PCR fragments from all three loci was obtained for 43 samples. Four of these samples were excluded from the analysis because they contained single gene typing fragments assigned to different assemblages (see above) and were most likely reflecting mixed infections. Thus, for 39 samples sequences of the individual typing fragments were first trimmed to a common length and then concatenated in the gene order ${ }^{5}-t p i-b g-g d h^{3^{\prime}}$ to yield a total sequence length of $1358 \mathrm{bp}$. These concatenated sequences and reference concatenated sequences representing assemblage AI, AII, BIII, BIV, and E types (see Additional file 1: Table S1 for accession numbers of the respective type sequences) were subsequently aligned. Comparisons were then performed to derive the number of common or different base pairs for each concatenated sequence pair. This revealed that only two sets of sequences were 100\% identical. Both were observed within the group of $\mathrm{E}$ type sequences, one representing two and the other representing four independent samples. Therefore, 35 unique concatenated sequencing types (2 A-type, 8 B-type, and 25 E-type; Additional file 2: Table S2) were available for further analysis.

Analysis of the pairwise comparisons of unique sequences belonging to the two larger groups showed that B-type sequences differed in 12 to 18 residues over their length of $1358 \mathrm{bp}$ from the chosen reference BIII and BIV fragments (Figure 1A). For E type sequences, this range was $8-17$ residues different to the reference E fragments (deduced from respective genes of the sequenced isolate P15; Figure 1B). Within the groups of Ismailia samples, B sequencing types differed on average in 14 residues and $E$ types in 6 residues. These values are consistent with the notion that intra-assemblage heterogeneity of $\mathrm{B}$ type isolates is higher than that of $\mathrm{E}$ or $\mathrm{A}$ type parasites [17].

Data on isolate genetic relatedness was integrated next with information on the geographic origin of the samples and projected onto a map of Ismailia province (Figure 2). Samples containing parasite DNA that was characterized by either an identical or near identical (1 of 1358 bp different) concatenated sequencing type were spatially related in 4 of 5 cases. The two human samples with a B sequencing type differing in a single base pair (sample pair number H66 and H90) were collected from children living in the same locality (Figure 2A). Similarly, all but one group of animal samples that contained $\mathrm{E}$ sequencing types that were genetic pairs (i.e. showed complete sequence identity or differed by only one base pair) were also geographic neighbors (Figure 2B; Clusters 1, 3, and 4). The distance between sample pairs that belonged to either of these assemblage B or E clusters was significantly shorter from the average distance calculated for all possible sample pairs (1.6 km vs 27.5 km; p < 0.01; two sided Student's T Test). Moreover, two of the samples belonging to cluster 2 (representing 5 samples, 4 with identical sequencing type and one with a single base pair difference; Figure $2 \mathrm{~B}$ ) originated from the same herd. Another one, $40 \mathrm{~b} / \mathrm{C} 7$, differed in only 3 base pairs from sample $40 \mathrm{c} / \mathrm{C} 10$ - the geographic neighbor, but displayed on average $7 \mathrm{bp}$ difference with the sequencing types of other samples. Therefore, sequence information, regarding even just these three typing loci, provided a high enough resolution to identify subpopulations of parasites that seemed to be linked epidemiologically.

\section{Discussion}

Understanding the epidemiology of giardiasis and estimating the zoonotic potential of its etiologic agent is necessary for rational disease management, particularly in regions of high prevalence in animals and/or humans. 


\begin{tabular}{|c|c|c|c|c|c|c|c|c|c|c|}
\hline & BIII & BIV & $\mathrm{H} 90$ & $\mathrm{H} 43$ & $\mathrm{H} 66$ & $\mathrm{H} 150$ & $\mathrm{H} 68$ & H88 & $\mathrm{H} 63$ & $\mathrm{H} 79$ \\
\hline BIII & $>$ & 18 & 17 & 17 & 16 & 16 & 15 & 14 & 14 & 13 \\
\hline BIV & 18 & $>$ & 16 & 14 & 16 & 12 & 18 & 18 & 15 & 12 \\
\hline H9O & 17 & 16 & $><$ & 18 & 1 & 16 & 12 & 19 & 18 & 10 \\
\hline $\mathrm{H} 43$ & 17 & 14 & 18 & $>$ & 18 & 15 & 18 & 18 & 13 & 13 \\
\hline H66 & 16 & 16 & 1 & 18 & $>$ & 16 & 11 & 19 & 18 & 10 \\
\hline $\mathrm{H} 150$ & 16 & 12 & 16 & 15 & 16 & $>1$ & 15 & 15 & 14 & 11 \\
\hline H68 & 15 & 18 & 12 & 18 & 11 & 15 & $>$ & 11 & 17 & 11 \\
\hline H88 & 14 & 18 & 19 & 18 & 19 & 15 & 11 & $>$ & 13 & 13 \\
\hline $\mathrm{H} 63$ & 14 & 15 & 18 & 13 & 18 & 14 & 17 & 13 & $>$ & 10 \\
\hline H79 & 13 & 12 & 10 & 13 & 10 & 11 & 11 & 13 & 10 & $>$ \\
\hline
\end{tabular}

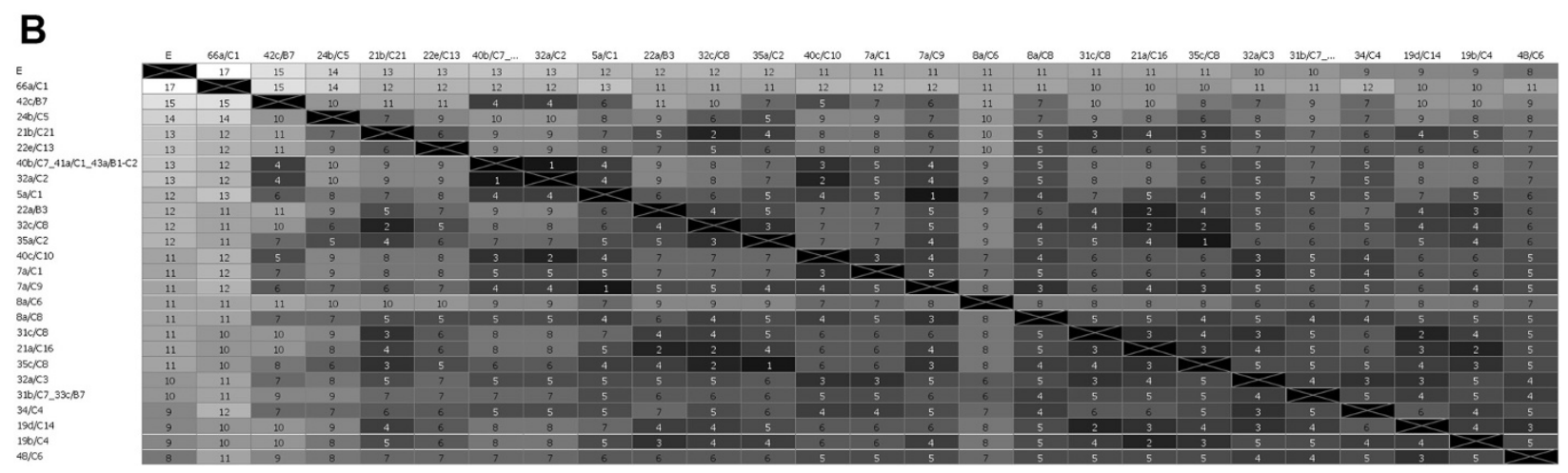

Figure 1 Relatedness of assemblage B and E sequencing types using distance matrix analysis. The sequencing fragments of samples with the complete information at all three gene loci were concatenated in the order of the tpi-bg-gdh sequences. The resulting sequencing fragments (1358 bp) were subsequently aligned using ClustalW. Shown are the deduced distance matrices for assemblage B (A) and assemblage E (B) sequencing types. Numbers and heatmap indicate nucleotide residues not identical between two sequences. We revealed only two complete type A sequences and these were excluded from the analysis. (A) Eight unique type B sequences could be analyzed. (B) 25 unique sequences (of a total of 29 type E sequences) were retrieved and analyzed. Samples $31 \mathrm{~b} / \mathrm{C} 7$ and 33c/B7 were identical as well as samples 40b/C7, 41a/C1, $43 \mathrm{a} / \mathrm{B} 1$ and $43 \mathrm{a} / \mathrm{C} 2$.

In this study the epidemiology of G. duodenalis infection in Ismailia province, Egypt was investigated. Previous studies on Giardia infections in Egypt and the Middle East have concentrated on detecting and analyzing human infections [31-39]. Depending on the study, a prevalence of $10-40 \%$ was reported. Here, this knowledge is complemented by providing prevalence estimates, genetic characterization and spatial distribution data of G. duodenalis assemblages detectable in samples from diarrheic children and animals from this geographic area. Overall prevalence in livestock was $53 \%$, while G. duodenalis was found in one of five children with diarrheal symptoms. While our typing results confirmed previous reports $[13,40]$ that zoonotic transmission from farm animals can occur, they also show that this is an infrequent event, even in an area of high Giardia prevalence in livestock and presumably low hygiene conditions. Consistent with this result, it was found that drinking tap water but not contact with animals was associated with increased risk for children to contract the infection. Thus, a dominant anthropogenic cycle is assumed that may be fuelled by low microbiological quality of water supply. Improving the latter will therefore be most likely efficient in reducing the burden of human infection in these sites.
The prevalence of $21 \%$ of Giardia infection in children with diarrheal symptoms sampled here was unexpectedly low. A report by Mahmoud et al. on G. duodenalis infection in infants from Bilbeis (a neighboring locality of our study area) showed that during the first year of life a child contracts on average 4.5 infections that last on average 7.2 weeks [41]. Based on this, a prevalence of 60-70\% was expected in children. Mahmud et al. also noted that children with diarrhea are much less likely to be tested positive for G. duodenalis, however, they did not elaborate on this in their report. Using the figures from the latter report for the diarrheal subgroup, a prevalence of $25 \%$ can be calculated, i.e. a number close to the $21 \%$ observed in the symptomatic group reported here. Data from at least three other studies suggest an inverse correlation between $G$. duodenalis infection in children and diarrheal symptoms $[5,42,43]$. This may be explained by a protective effect of Giardia colonization against unrelated causes of diarrhea; alternatively it may reflect a lower rate of parasite detection in symptomatic cases if diarrhea is viewed as a response of the host organism to reduce parasite burden.

The Bilbeis study [41] as well as others [5,44,45], also suggested that breast feeding has a preventive or protective 


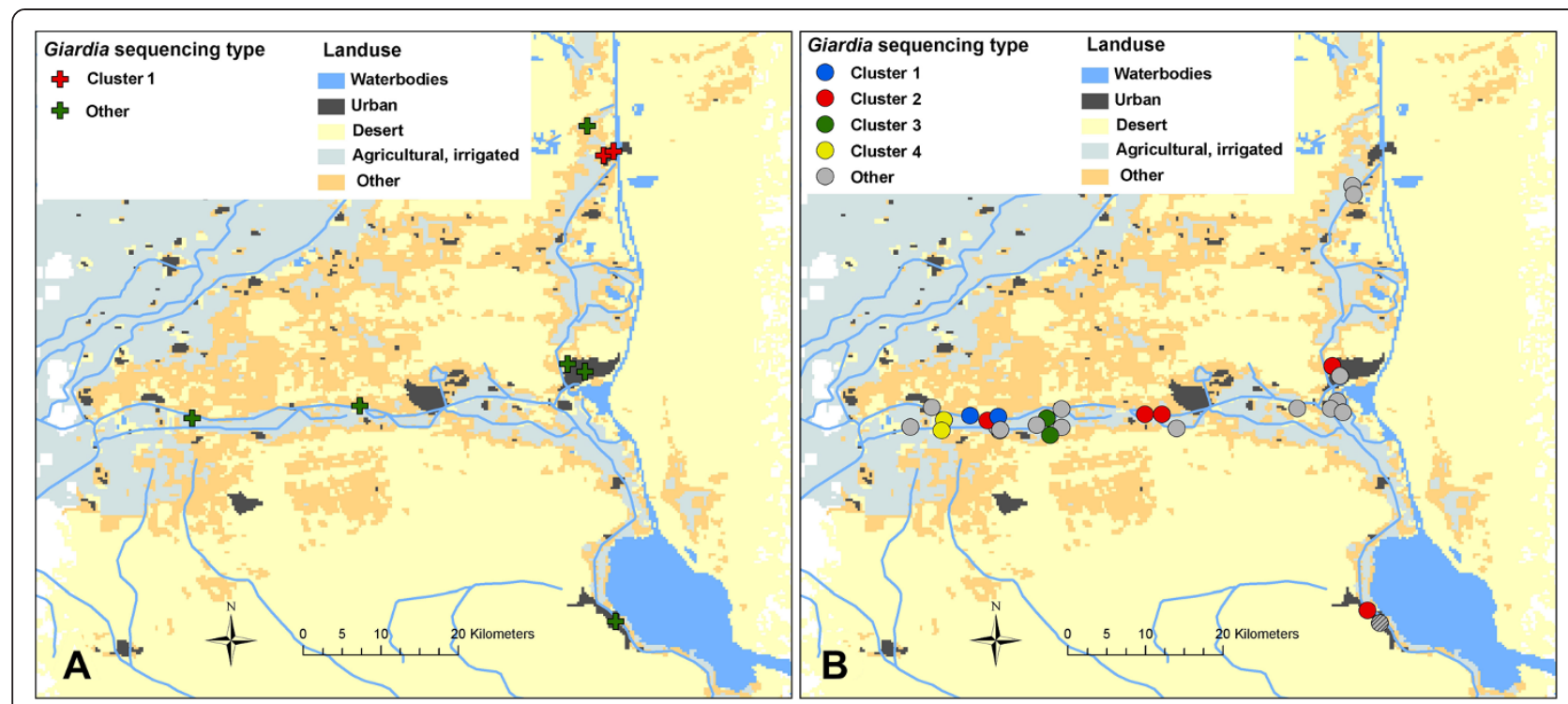

Figure 2 Spatial distribution of samples containing assemblage B and E type parasites. Clusters of concatenated B and E type sequences were defined based on sequence identity, i.e. sequences that were identical or that differed in maximally one base pair were combined in one cluster. The spatial distribution of sequence types was visualized on a map of the study region. (A) Analysis of the 8 B type sequences revealed one cluster with 2 sequences that differed in one base pair (samples H66 and H90). (B) Analysis of 29 E type sequences revealed 4 clusters. Cluster 1 comprising of the identical sequences of samples $31 \mathrm{~b} / \mathrm{C} 7$ and $33 \mathrm{C} / \mathrm{B} 7$. Cluster 2 comprising of the 4 identical sequences of samples $40 \mathrm{~b} / \mathrm{C} 7,41 \mathrm{a} / \mathrm{C} 1,43 \mathrm{a} / \mathrm{B} 1$ and $43 \mathrm{a} / \mathrm{C} 25$, and of sequence of sample $32 \mathrm{a} / \mathrm{C} 2$ that differed in one base pair. Cluster 3 comprising of sequences of samples $5 \mathrm{a} / \mathrm{C} 1$ and $7 \mathrm{a} / \mathrm{C} 9$ that differ in one base pair. Cluster 4 comprising of sequences of samples $35 \mathrm{a} / \mathrm{C} 2$ and $35 \mathrm{c} / \mathrm{C} 8$ that differ in one base pair. Note that sample 40c/C10 (shaded grey area) differed only in three base pairs from sample 40b/C7 of cluster 2.

effect against G. duodenalis infection. Thus, one would expect to detect lower parasite burdens in breast-fed infants. This is corroborated by the trend that was observed in the pediatric population investigated here. Children below 1 year of age, which in this area were likely to be breast-fed, displayed a higher prevalence based on real-time PCR but lower abundance of G. duodenalis compared to older children. Future work will have to substantiate this observation.

Gastrointestinal infections may predispose for coinfections. A possible positive association of Cryptosporidium and Giardia infections has been reported in some earlier studies [46-48], but most reports investigating possible co-infections with these two parasites did not find such an association [49-51]. In the present study a positive association between Giardia and Cryptosporidium infections in ruminants was observed; this may be due to a high rate of concomitant contamination of the water resources with both parasites.

Molecular typing of pathogens has enabled highly informative insight into the epidemiology of many infections [52]. Implementation of nucleic acid-based detection and typing methods over the past decade has led researchers to better understand the complexity of the Giardia genus including the parasite population relevant to human and veterinary health $[15,18]$. Before discussing our findings in this regard, some general comments about interpreting typing data in this field are warranted as this remains difficult [18].

Firstly, the parasites have two equivalent nuclei with full diploid chromosomal content and a single parasite could theoretically present up to four alleles of a single copy gene. Since parasites of only a minority of assemblages can be cultured and cloned with reasonable success, typing for the majority of samples relies on DNA prepared from cysts and typing of single cysts is currently not a realistic option. As a consequence, "mixed" sequencing results cannot unambiguously be assigned to mixed infection (which seems more probable in case of inter-assemblage ambiguities; cf. $[16,18]$ ) rather than to infection with heterozygotic parasites (more likely in case of "double peaks" and intra-assemblage ambiguities). Secondly, current PCR-based locus-specific typing assays show significant differences in sensitivity and bias in efficiency of amplifying a given assemblage [18]. This was also observed in our study. For example, locus-directed, assemblage A and E specific PCR assays displayed a lower sensitivity than the non-assemblage-specific assays and the tpi locus directed general PCR assay likely introduced a typing bias such that assemblage A type sequences were more readily detected. Out of 11 animal samples, which were typed as assemblage A, only two could be typed at other loci and, when successful, these were identified as type E amplicons. Re-testing these two samples with assemblage A 
and E-specific tpi-directed assays showed the same result. In view of all these caveats, we interpret the obtained typing data as providing operational rather than categorical classification of the parasite DNA.

In agreement with others [16], all sequencing types with assignments to more than one assemblage were considered as the result of mixed infections. Typically these typings were less robust either because fragments of only one locus could be amplified or because repeated analyses using more assemblage-specific typing assays failed possibly due to the lower sensitivity. In contrast, typing results showing intra-assemblage ambiguities were much more reliable, i.e. they were reproducible within a sample and identical patterns were repeatedly observed in independent samples. Although sexual recombination in Giardia may occur [53-55], at the population level this is thought to be rare and mostly to involve selfing [16]. Hence, it is reasonable to assume that particular sequencing types with data available for all three loci assigned to only one assemblage reflect distinct, identifiable and traceable isolates. Consistent with this, a closely related sequencing type was a good predictor for a similar geographic origin. Therefore, it is proposed that at least for assemblages B or E, intra-assemblage sequence differences are large enough to allow detection of epidemiological links even with the currently most widely used typing scheme that is based on just three loci. Consequently, infections due to parasites with identical typing fragments but for which respective samples were collected from hosts living more distantly from each other may therefore be linked by factors that had not been recorded here, such as animal, food or manure trading.

\section{Conclusion}

G. duodenalis in Ismailia province was commonly found in livestock and children. Infection and diarrheal symptoms were not positively correlated; rather this relationship was inversed in children. Findings from genetic characterization indicate that infection cycles in humans and ruminants are largely separated. Thus, the risk of zoonotic infection emanating from cattle and buffalo is negligible from an epidemiological point of view. Distinct circulating parasite subpopulations were traceable by sequence typing. This suggested that the methodology is suitable for epidemiological studies on occurrence and spreading of G. duodenalis in humans and animals. This may also prove useful to monitor the effects of strategies that aim at interfering with transmission.

\section{Additional files}

Additional file 1: Analysis of heterozygous sequences and ambiguous single nucleotide polymorphisms.
Additional file 2: Summary of genetic characterization of Giardia samples by PCR.

\section{Competing interests}

The authors declare that they have no competing interests.

\section{Authors' contributions}

YAH performed the experiments, analyzed the data, and wrote the manuscript; CK and TA designed and supervised the study, analyzed the data and wrote the manuscript; HW perfomed the spatial distribution analysis; JK, KN, and GVH contributed reagents and wrote the manuscript; $\mathrm{KHZ}$ performed the initial study desing for sample collection and wrote the manuscript. All authors read and approved the final version of the manuscript.

\section{Acknowledgement}

We thank the R-biopharm AG, Darmstadt, for providing us with the RIDA® QUICK test for the immunological chair and fecal examinations. We thank Prof. Dr. Rabei Saleh, Prof. Dr. Abdelkareem Abou-Eisha, Prof. Dr. Mohamed Sobieh, Dr. Ahmed Abdel-Maksoud and Dr. Ahmed Sobieh, for their help during samples collection. We thank Petra Gosten-Heinrich and Elke Radam for their help in the lab. We thank Noelle O'Regan for proofreading. The German Academic Exchange Service (DAAD) and the Ministry of Higher Education in Egypt allocated funds for the study.

\section{Author details}

${ }^{1}$ Department of Animal Hygiene, Zoonoses and Animal Ethology, Faculty of Veterinary Medicine, Suez Canal University, 41511 Ismailia, Egypt. ${ }^{2}$ Faculty Panel Veterinary Public Health, Freie Universität Berlin, 14163 Berlin, Germany. ${ }^{3}$ Mycotic and parasitic agents and mycobacteria, Department of Infectious Diseases, Robert Koch-Institute, 13353 Berlin, Germany. ${ }^{4}$ Gastrointestinal Infections, Zoonoses and Tropical Infections, Department of Infectious Disease Epidemiology, Robert Koch-Institute, 13353 Berlin, Germany. ${ }^{5}$ Institute for Parasitology and Tropical Veterinary Medicine, Freie Universität Berlin, 14163 Berlin, Germany. ${ }^{6}$ Federal Institute for Risk Assessment (BfR), 12277 Berlin, Germany.

Received: 15 January 2014 Accepted: 5 July 2014

Published: 11 July 2014

\section{References}

1. Savioli L, Smith H, Thompson A: Giardia and Cryptosporidium join the 'Neglected Diseases Initiative'. Trends Parasito/ 2006, 22(5):203-208.

2. Halliez MC, Buret AG: Extra-intestinal and long term consequences of Giardia duodenalis infections. World journal of gastroenterology. World J Gastroenterol 2013, 19(47):8974-8985.

3. Gardner TB, Hill DR: Treatment of giardiasis. Clin Microbiol Rev 2001, 14(1):114-128

4. Feng Y, Xiao L: Zoonotic potential and molecular epidemiology of Giardia species and giardiasis. Clin Microbiol Rev 2011, 24(1):110-140.

5. Ignatius R, Gahutu JB, Klotz C, Steininger C, Shyirambere C, Lyng M, Musemakweri A, Aebischer T, Martus P, Harms G, Mockenhaupt FP: High prevalence of Giardia duodenalis Assemblage B infection and association with underweight in Rwandan children. PLoS Neglect Trop 2012, 6(6):e1677.

6. Caccio SM, Ryan U: Molecular epidemiology of giardiasis. Mol Biochem Parasitol 2008, 160(2):75-80.

7. Ignatius R, Gahutu JB, Klotz C, Musemakweri A, Aebischer T, Mockenhaupt FP. Detection of Giardia duodenalis assemblage A and B isolates by immunochromatography in stool samples from Rwandan children. Clin Microbiol Infect 2014, doi:10.1111/1469-0691.12596. [Epub ahead of print].

8. Mank TG, Zaat JO, Deelder AM, Van Eijk JT, Polderman AM: Sensitivity of microscopy versus enzyme immunoassay in the laboratory diagnosis of giardiasis. Eur J Clin Microbiol Infect Dis 1997, 16(8):615-619.

9. Verweij JJ, Schinkel J, Laeijendecker D, Van Rooyen MA, Van Lieshout L, Polderman AM: Real-time PCR for the detection of Giardia lamblia. Mol Cell Probes 2003, 17(5):223-225.

10. David EB, Coradi ST, Oliveira-Sequeira TCG, Ribolla PEM, Katagiri S, Guimarães S: Diagnosis of Giardia infections by PCR-based methods in children of an endemic area. J Venom Anim Toxins Trop Dis 2011, 17(2):209-215. 
11. Monis PT, Caccio SM, Thompson RC: Variation in Giardia: towards a taxonomic revision of the genus. Trends Parasitol 2009, 25(2):93-100.

12. Read CM, Monis PT, Thompson RC: Discrimination of all genotypes of Giardia duodenalis at the glutamate dehydrogenase locus using PCR-RFLP. Infect Genet Evol 2004, 4(2):125-130

13. Sulaiman IM, Fayer R, Bern C, Gilman RH, Trout JM, Schantz PM, Das P, Lal $A A$, Xiao L: Triosephosphate isomerase gene characterization and potential zoonotic transmission of Giardia duodenalis. Emerg Infect Dis 2003, 9(11):1444-1452.

14. Lalle M, Pozio E, Capelli G, Bruschi F, Crotti D, Cacciò SM: Genetic heterogeneity at the beta-giardin locus among human and animal isolates of Giardia duodenalis and identification of potentially zoonotic subgenotypes. Int J Parasitol 2005, 35(2):207-213.

15. Thompson RC, Monis P: Giardia-from genome to proteome. Adv Parasitol 2012, 78:57-95.

16. Takumi K, Swart A, Mank T, Lasek-Nesselquist E, Lebbad M, Caccio SM, Sprong H: Population-based analyses of Giardia duodenalis is consistent with the clonal assemblage structure. Parasit Vectors 2012, 5:168

17. Caccio SM, Sprong H: Giardia duodenalis: genetic recombination and its implications for taxonomy and molecular epidemiology. Exp Parasitol 2010, 124(1):107-112.

18. Ryan U, Caccio SM: Zoonotic potential of Giardia. Int J Parasitol 2013, 43(12-13):943-956.

19. Thompson RC: Giardiasis as a re-emerging infectious disease and its zoonotic potential. Int J Parasitol 2000, 30(12-13):1259-1267.

20. Helmy YA, Krücken J, Nöckler K, Von Samson-Himmelstjerna G, Zessin K-H: Molecular epidemiology of Cryptosporidium in livestock animals and humans in the Ismailia province of Egypt. Vet Parasito/ 2013, 193(1-3):15-24

21. Lindley DV, Scott WF: New Cambridge Elementary Statistical Tables. Cambridge: Cambridge Unviversity press; 1984

22. Verweij JJ, Blange RA, Templeton K, Schinkel J, Brienen EA, Van Rooyen MA, Van Lieshout L, Polderman AM: Simultaneous detection of Entamoeba histolytica, Giardia lamblia, and Cryptosporidium parvum in fecal samples by using multiplex real-time PCR. J Clin Microbiol 2004, 42(3):1220-1223.

23. Caccio SM, De Giacomo M, Pozio E: Sequence analysis of the beta-giardin gene and development of a polymerase chain reaction-restriction fragment length polymorphism assay to genotype Giardia duodenalis cysts from human faecal samples. Int J Parasitol 2002, 32(8):1023-1030.

24. Geurden T, Geldhof P, Levecke B, Martens C, Berkvens D, Casaert S, Vercruysse J, Claerebout E: Mixed Giardia duodenalis assemblage A and E infections in calves. Int J Parasitol 2008, 38(2):259-264.

25. Sprong $\mathrm{H}$, Caccio SM, van der Giessen JW, network Z, partners: Identification of zoonotic genotypes of Giardia duodenalis. PLoS Neglect Trop 2009, 3(12):e558.

26. Edgar RC: MUSCLE: multiple sequence alignment with high accuracy and high throughput. Nucleic Acids Res 2004, 32(5):1792-1797.

27. Weitzel T, Dittrich S, Mohl I, Adusu E, Jelinek T: Evaluation of seven commercial antigen detection tests for Giardia and Cryptosporidium in stool samples. Clin Microbiol Infect 2006, 12(7):656-659.

28. Sabry MA, Taher ES, Meabed EMH: Prevalence and Genotyping of Zoonotic Giardia from Fayoum Governorate, Egypt. Res J Parasitol 2009, 4:105-114.

29. Monis PT, Andrews RH, Mayrhofer G, Ey PL: Genetic diversity within the morphological species Giardia intestinalis and its relationship to host origin. Infect Genet Evol 2003, 3(1):29-38.

30. Linz B, Balloux F, Moodley Y, Manica A, Liu H, Roumagnac P, Falush D, Stamer C, Prugnolle F, van der Merwe SW, Yamaoka Y, Graham DY, Perez-Trallero E, Wadstrom T, Suerbaum S, Achtman M: An African origin for the intimate association between humans and Helicobacter pylori. Nature 2007 445(7130):915-918.

31. Mohamed NH, Salama MM, Moustafa MA, El-Wakil HS, Mohareb EW, Thabet HS: Molecular characterization of Egyptian Giardia lamblia isolates. J Egypt Soc Parasitol 2004, 34(1):213-226.

32. Foronda P, Bargues MD, Abreu-Acosta N, Periago MV, Valero MA, Valladares B, Mas-Coma S: Identification of genotypes of Giardia intestinalis of human isolates in Egypt. Parasitol Res 2008, 103(5):1177-1181.

33. Helmy MM, Abdel-Fattah HS, Rashed L: Real-time PCR/RFLP assay to detect Giardia intestinalis genotypes in human isolates with diarrhea in Egypt. J Parasitol 2009, 95(4):1000-1004.

34. Shukry S, Zaki AM, DuPont HL, Shoukry I, El Tagi M, Hamed Z: Detection of enteropathogens in fatal and potentially fatal diarrhea in Cairo, Egypt. J Clin Microbiol 1986, 24(6):959-962.
35. Fawzi M, El-Sahn AA, Ibrahim HF, Shehata Al: Vegetable-transmitted parasites among inhabitants of El-Prince, Alexandria and its relation to housewives' knowledge and practices. J Egypt Public Health Assoc 2004, 79(1-2):13-29.

36. Curtale F, Nabil M, El Wakeel A, Shamy MY: Anaemia and intestinal parasitic infections among school age children in Behera Governorate, Egypt. Behera Survey Team. J Trop Pediatr 1998, 44(6):323-328.

37. Shalaby I, Gherbawy Y, Banaja A: Molecular characterization of Giardia parasite isolated from stool samples collected from different hospitals in Taif City (Saudi Arabia). Trop Biomed 2011, 28(3):487-496.

38. Amer SE: Genotypic and phylogenetic characterization of Giardia intestinalis from human and dairy cattle in Kafr El Sheikh Governorate, Egypt. J Egypt Soc Parasitol 2013, 43(1):133-146.

39. Nazeer JT, El Sayed KK, Von Thien H, El-Sibaei MM, Abdel-Hamid MY, Tawfik RA, Tannich E: Use of multiplex real-time PCR for detection of common diarrhea causing protozoan parasites in Egypt. Parasitol Res 2013, 112(2):595-601.

40. Santin M, Trout JM, Fayer R: Prevalence and molecular characterization of Cryptosporidium and Giardia species and genotypes in sheep in Maryland. Vet Parasitol 2007, 146(1-2):17-24.

41. Mahmud MA, Chappell CL, Hossain MM, Huang DB, Habib M, DuPont HL: Impact of breast-feeding on Giardia lamblia infections in Bilbeis, Egypt. Am J Trop Med Hyg 2001, 65(3):257-260.

42. Veenemans J, Mank T, Ottenhof M, Baidjoe A, Mbugi EV, Demir AY, Wielders JP, Savelkoul HF, Verhoef H: Protection against diarrhea associated with Giardia intestinalis is lost with multi-nutrient supplementation: a study in Tanzanian children. PLoS Negl Trop Dis 2011, 5(6):e1158.

43. Bodhidatta L, McDaniel P, Sornsakrin S, Srijan A, Serichantalergs O, Mason $\mathrm{CJ}$ : Case-control study of diarrheal disease etiology in a remote rural area in Western Thailand. Am J Trop Med Hyg 2010, 83(5):1106-1109.

44. Morrow AL, Reves RR, West MS, Guerrero ML, Ruiz-Palacios GM, Pickering LK: Protection against infection with Giardia lamblia by breast-feeding in a cohort of Mexican infants. J Pediatr 1992, 121(3):363-370.

45. Lunn PG, Erinoso HO, Northrop-Clewes CA, Boyce SA: Giardia intestinalis is unlikely to be a major cause of the poor growth of rural Gambian infants. J Nutr 1999, 129(4):872-877.

46. Wolfson JS, Hopkins CC, Weber DJ, Richter JM, Waldron MA, McCarthy DM: An association between Cryptosporidium and Giardia in stool. N Engl J Med 1984, 310(12):788

47. Jokipii L, Pohjola S, Jokipii AM: Cryptosporidiosis associated with traveling and giardiasis. Gastroenterol 1985, 89(4):838-842.

48. Wang L, Xiao L, Duan L, Ye J, Guo Y, Guo M, Liu L, Feng Y: Concurrent Infections of Giardia duodenalis, Enterocytozoon bieneusi, and Clostridium difficile in Children during a Cryptosporidiosis Outbreak in a Pediatric Hospital in China. PLoS Negl Trop Dis 2013, 7(9):e2437.

49. Rinaldi L, Musella V, Condoleo R, Saralli G, Veneziano V, Bruni G, Condoleo RU, Cringoli G: Giardia and Cryptosporidium in water buffaloes (Bubalus bubalis). Parasitol Res 2007, 100(5):1113-1118.

50. Skeels MR, Sokolow R, Hubbard CV, Foster LR: Screening for coinfection with Cryptosporidium and Giardia in Oregon public health clinic patients. Am J Public Health 1986, 76(3):270-273.

51. Becher KA, Robertson ID, Fraser DM, Palmer DG, Thompson RC: Molecular epidemiology of Giardia and Cryptosporidium infections in dairy calves originating from three sources in Western Australia. Vet Parasitol 2004, 123(1-2):1-9.

52. Achtman M: Evolution, population structure, and phylogeography of genetically monomorphic bacterial pathogens. Annu Rev Microbiol 2008, 62:53-70.

53. Cooper MA, Adam RD, Worobey M, Sterling CR: Population genetics provides evidence for recombination in Giardia. Curr Biol 2007, 17(22):1984-1988.

54. Poxleitner MK, Dawson SC, Cande WZ: Cell cycle synchrony in Giardia intestinalis cultures achieved by using nocodazole and aphidicolin. Eukaryot Cell 2008, 7(4):569-574.

55. Lasek-Nesselquist E, Welch DM, Thompson RC, Steuart RF, Sogin ML: Genetic exchange within and between assemblages of Giardia duodenalis. J Eukaryot Microbiol 2009, 56(6):504-518.

doi:10.1186/1756-3305-7-321

Cite this article as: Helmy et al:: Epidemiology of Giardia duodenalis infection in ruminant livestock and children in the Ismailia province of Egypt: insights by genetic characterization. Parasites \& Vectors 2014 7:321. 